\title{
Preventing congenital varicella syndrome with immunization
}

\author{
Andreas Sauerbrei MD
}

See related review article by Cohen and colleagues, previously published at www.cmaj.ca

$\mathrm{C}$ ongenital varicella syndrome is a rare complication of infection with maternal varicella before 20 weeks of pregnancy. Prospective studies in Europe and North America have shown that the incidence of congenital varicella syndrome in maternal varicella contracted in the first 20 weeks of pregnancy is about $1 \%-2 \% .^{1,2}$ Based on this estimated risk (1\%), on an estimated risk of varicella infection during pregnancy of two maternal infections per 1000 pregnancies and on the annual birth rate, the number of expected cases of congenital varicella syndrome per year is approximately 41 in the United States, 4 in Canada, 7 in the United Kingdom and 7 in Germany. ${ }^{3}$ This congenital infection may be associated with severe anomalies, such as limb hypoplasia, neurologic defects, eye diseases and skin lesions. Nearly $30 \%$ of infants born with signs of congenital varicella syndrome die during the first few months of life. ${ }^{4}$

Numerous reviews have underlined the possibility of preventing congenital varicella syndrome using varicella vaccination or passive immunization. Nevertheless, the syndrome has to be considered a worldwide unsolved medical problem to date. About 150 case reports of congenital varicella syndrome have been published in the English and German literatures, with three listed in the PubMed database of the National Library of Medicine in 2010. These data are a reminder for all health professionals who counsel women who have been in contact with chickenpox early in pregnancy to keep in mind the potential risks and permanent consequences of possible fetal infection with varicella-zoster virus.

\section{Varicella vaccination}

Effective prophylaxis (i.e., primary prevention) of chickenpox and associated fetal infection in pregnant women is possible only by active immunization of seronegative women before pregnancy. Varicella vaccines, based on a liveattenuated Oka strain, are available in most countries. Although the varicella vaccine is con- traindicated in pregnant women (as are all liveattenuated vaccines), inadvertent vaccination during pregnancy does not appear to result in an increase in incidence of congenital varicella syndrome or other birth defects. ${ }^{5}$ In the case of congenital varicella syndrome presented by Cohen and colleagues, ${ }^{6}$ the crucial failure was the omission of vaccination for varicella before pregnancy after serologic testing had revealed a lack of immunity in the mother.

\section{Varicella-zoster immune globulin}

There are no controlled clinical trials examining the efficacy of varicella-zoster immune globulin, also known as VZIG, in healthy adults, infants or neonates. Studies involving immunocompromised patients show that the severity of clinical disease and its complications can be reduced by 75\%. ${ }^{7}$ The risks associated with passive immunization with varicella-zoster immune globulin are minimal. ${ }^{8}$

If an unvaccinated pregnant woman with a negative or indeterminate history of varicella has been exposed to varicella-zoster virus by household contact, face-to-face contact for at least 5 minutes or indoor contact for more than 15 minutes, virus-specific IgG antibodies should be measured without delay. In instances of negative, indeterminate or unknown serologic status, the administration of varicella-zoster immune globulin within 96 hours is strongly indicated. This procedure for secondary prevention of varicella is in accordance with German ${ }^{9}$
Competing interests: None declared.

This article was solicited and has not been peer reviewed.

Correspondence to: Dr. Andreas Sauerbrei, andreas.sauerbrei@ med.uni-jena.de

CMAJ 2011. DOI:10.1503 /cmaj.110055

\section{KEY POINTS}

- The most effective way to prevent varicella and associated fetal infection in a seronegative mother is immunization with varicella vaccine before pregnancy.

- Administration of varicella-zoster immune globulin (VZIG) to a seronegative woman exposed to varicella in pregnancy can prevent severe maternal infection and its complications.

- Although administration of VZIG to a seronegative pregnant woman exposed to varicella before 20 weeks of pregnancy cannot eliminate the risk of fetal infection, it represents the only chance to prevent the severe consequences of congenital varicella syndrome. 
and British guidelines ${ }^{10}$ for prevention of chickenpox in pregnancy, even though the specific timing of administration of varicella-zoster immune globulin is controversial. The evidence that varicella-zoster immune globulin prevents or attenuates varicella in pregnancy comes from a prospective study by Enders and coauthors. ${ }^{1,11}$ Their study group, however, was too small to show an elimination of risk of fetal infection. Hence, the primary reason for the administration of varicella-zoster immune globulin is to prevent severe maternal chickenpox and its complications (e.g., pneumonia).

The risk of fetal infection may be reduced with timely administration of varicella-zoster immune globulin. This is supported by welldesigned cohort studies, which were analyzed by Cohen and colleagues. ${ }^{6}$ However, the administration of varicella-zoster immune globulin has not been found to reliably prevent viremia, which is associated with clinically apparent varicella. In the study by Enders and colleagues, ${ }^{1}$ about half of the mothers developed clinical varicella even when varicella-zoster immune globulin was given within one to three days of substantive exposure. Therefore, it can be assumed that varicella-zoster immune globulin may lessen, but does not eliminate, the viral load to which the fetus is exposed. Pastuszak and colleagues ${ }^{2}$ reported an instance of congenital varicella syndrome that occurred despite receipt by the mother of varicella-zoster immune globulin four days postexposure.

\section{Clinical practice guidelines}

What is the difference between practice guidelines for prevention of chickenpox during pregnancy in North America and the European guidelines? As described by Cohen and coauthors, ${ }^{6}$ several North American guidelines do not strongly recommend varicella-zoster immune globulin in pregnant women and do not consider the possibility that passive immunization may reduce the risk of fetal infection, in contrast to the British ${ }^{8}$ and German guidelines. ${ }^{10}$ Nevertheless, passive immunization with varicella-zoster immune globulin represents the only chance to prevent the severe consequences of congenital varicella syndrome if a seronegative pregnant woman is exposed to varicella-zoster virus before 20 weeks of pregnancy. Chemoprophylaxis using acyclovir, valacyclovir or famciclovir has not been proven safe during early pregnancy.

The administration of varicella-zoster immune globulin should be strongly recommended in seronegative pregnant women who come into contact with varicella, and its relatively high costs should not deter physicians from its use.

\section{References}

1. Enders G, Miller E, Cradock-Watson J, et al. Consequences of varicella and herpes zoster in pregnancy: prospective study of 1739 cases. Lancet 1994;343:1548-51

2. Pastuszak AL, Levy M, Schick B, et al. Outcome after maternal varicella infection in the first 20 weeks of pregnancy. $N$ Engl J Med 1994;330:901-5.

3. Tan MP, Koren G. Chickenpox in pregnancy: revisited. Reprod Toxicol 2006;21:410-20.

4. Sauerbrei A, Wutzler P. The congenital varicella syndrome. $J$ Perinatol 2000;20:548-54.

5. Wilson E, Goss MA, Marin M, et al. Varicella vaccine exposure during pregnancy: data from 10 years of the pregnancy registry. J Infect Dis 2008;197(Suppl 2):S178-84.

6. Cohen A, Maschopoulos P, Stiehm RE, et al. Congenital varicella syndrome: the evidence for secondary prevention with varicella-zoster immune globulin. CMAJ 2011;183:204-8.

7. Zaia JA, Levin MJ, Preblud SR, et al. Evaluation of varicella-zoster immune globulin: protection of immunosuppressed children after household exposure to varicella. J Infect Dis 1983;147:737-43.

8. Koren G, Money D, Boucher M, et al. Serum concentrations, efficacy, and safety of a new, intravenously administered varicella zoster immune globulin in pregnant women. J Clin Pharmacol 2002;42:267-74.

9. Robert Koch-Institut. Empfehlungen der Ständigen Impfkommission (STIKO) am Robert Koch Institut. Stand: Juli 2004. Epidemiologisches Bulletin 2004;235-50.

10. Royal College of Obstetricians and Gynaecologists. Green-top Guideline No. 13. Chickenpox in pregnancy. London (UK): The College; 2007. Available: www.rcog.org.uk/files/rcog-corp /uploaded-files/GT13ChickenpoxinPregnancy2007.pdf (accessed 2011 Jan. 4).

11. Enders G, Miller E. Varicella and herpes zoster in pregnancy and the newborn. In: Arvin AM, Gershon AA, editors. Varicella-zoster virus. Virology and clinical management. Cambridge (UK): University Press; 2000. p. 317-47.

Affiliation: Andreas Sauerbrei is with the German Reference Laboratory for HSV and VZV, Institute of Virology and Antiviral Therapy, Jena University Clinic, Jena, Germany. 